\title{
AUTORREGULAÇÃO DA APRENDIZAGEM E A CONSTRUÇÃO DE SOFTWARES EDUCACIONAIS: UM ESTUDO DE CASO
}

Rebeca L. S. Maia Gomes - reebecamaia@gmail.com

Tarso V. Ferreira - tarso@academico.ufs.br

Lucas T. J. Pereira - lucastjp@ dcomp.ufs.br

Almir C. dos Santos Júnior - almircarlossj@gmail.com

Universidade Federal de Sergipe, Departamento de Engenharia Elétrica

Avenida Marechal Rondon, S/N, Rosa Elze

49100-000 - São Cristóvão - Sergipe

Resumo: No contexto da educação em engenharia, é possível compreender a importância da utilização de recursos tecnológicos capazes de estimular a adoção de um modelo de ensino colaborativo, ativo, capaz de auxiliar os estudantes a desenvolver habilidades necessárias para obter sucesso profissional em uma sociedade em constante evolução. Entre as ferramentas educacionais tecnológicas, os jogos se destacam por serem capazes de beneficiar diversos aspectos do estudante, entre eles os aspectos afetivos, sociais e cognitivos, além de possibilitar o desenvolvimento de características como a imaginação, a imitação, a regra, tomada de decisões e o raciocínio lógico. Neste trabalho, propõe-se apresentar uma abordagem que relacione a teoria da autorregulação da aprendizagem aos princípios de aprendizagem incorporados pelos jogos. Espera-se que a abordagem proposta possa fornecer subsídios para o desenvolvimento de softwares educacionais interativos, atuantes no processo de aprendizagem, e que estimulem a adoção de motivações e estratégias relacionadas à aprendizagem profunda. Como estudo de caso, é apresentado o software educacional "Plugue", desenvolvido para o ensino de instalações elétricas prediais, que utilizou a abordagem proposta como eixo referencial para sua construção e sua forma de intervenção junto ao processo de aprendizagem.

Palavras-chave: Engenharia. Educação. Autorregulação da aprendizagem. Abordagens à Aprendizagem. Jogos educacionais.

\section{INTRODUÇÃO}

As constantes transformações sociais, políticas e tecnológicas têm ocasionado diversas mudanças no setor educacional, o qual tem exigido processos de aprendizagem cada vez mais focados na formação de competências relacionadas à criatividade, inovação, empreendedorismo, raciocínio lógico e resolução de problemas. No âmbito da engenharia, torna-se imprescindível o emprego de recursos tecnológicos nas salas de aula e fora delas, que auxiliem a migração de um modelo de transferência de conhecimento para um modelo colaborativo, ativo e autodirigido, e que auxilie os estudantes a desenvolver habilidades necessárias para obter sucesso profissional em uma sociedade em evolução.

Considerando que a tecnologia torna possível envolver os estudantes em atividades mais relevantes e autênticas, facilitando o aprendizado, ela pode ser utilizada também como uma ferramenta que viabiliza a autorregulação da aprendizagem por parte dos alunos. Entre as ferramentas educacionais tecnológicas, os jogos se destacam por serem capazes de beneficiar os aspectos afetivos, sociais e cognitivos do estudante, permitindo desenvolver características como a imaginação, a imitação, a regra, tomada de decisões e o raciocínio lógico. 
Os bons jogos incorporam alguns dos princípios de aprendizagem, os quais estão relacionados às fases da autorregulação da aprendizagem.

Sendo assim, a dinâmica por trás dos jogos pode auxiliar o estudante a tornar-se mais autorregulado ao estimular a elaboração de estratégias para o cumprimento de metas, ação baseada no planejamento e a autoavaliação, através de feedbacks e pontuações.

Neste trabalho, propõe-se apresentar uma abordagem que relacione a teoria da autorregulação da aprendizagem aos princípios de aprendizagem incorporados pelos games. Espera-se que a abordagem proposta possa fornecer subsídios para o desenvolvimento de softwares educacionais interativos, atuantes no processo de aprendizagem, e que estimulem a adoção de motivações e estratégias relacionadas à aprendizagem profunda, contribuindo para o desenvolvimento de aspectos cognitivos do estudante. Como estudo de caso, é apresentado o software educacional "Plugue" que utilizou a abordagem proposta como eixo referencial para sua construção e sua forma de intervenção junto ao processo de aprendizagem, e cuja temática consiste no ensino de instalações elétricas prediais.

\section{FUNDAMENTAÇÃO TEÓRICA}

Serão apresentados os principais conceitos relacionados à abordagens à aprendizagem, autorregulação da aprendizagem e jogos educacionais, por se tratarem de temas substancias para o desenvolvimento deste trabalho.

\subsection{Abordagens à aprendizagem}

Os alunos possuem diferentes motivações e estratégias para aprender (BIGGS, 1978). O estudo das diferentes abordagens à aprendizagem que os alunos adotam está relacionado com a participação do estudante na seleção, interpretação e aplicação do conhecimento acadêmico, enfatizando a interação ativa do estudante com os objetos de conhecimento, e se contrapõe aos modelos tradicionais, transmissivos, focados na reprodução das informações, nos quais o aluno é passivo no processo de aquisição do conhecimento (BORUCHOVITCH, 1999; DE CORTE, 2000).

Marton e Saljö (1976a, 1976b) foram os pioneiros nos estudos sobre abordagens à aprendizagem. Durante suas pesquisas, perceberam que estudantes apresentam diferentes padrões de leitura e compreensão de textos e chamaram essas diferenças de Abordagem Superficial (AS) e Abordagem Profunda (AP). Em sua definição, a AP envolve uma postura ativa do sujeito junto ao processo de aprendizagem, demarcando uma conexão entre motivação e estratégia ativas (BIGGS, 1987b). São características dessa abordagem a interpretação de aspectos explícitos e implícitos, a construção de relações amplas e abstratas, assim como a busca pela compreensão e formação de significados pessoais frente aos objetos de conhecimento. A AS envolve a relação entre uma motivação e as estratégias passivas de aprendizagem, e conduz a uma retenção de detalhes pontuais, por meio da memorização, o que dificulta e limita a interação entre o sujeito e o objeto de estudo (BIGGS, 1987a; ENTWISTLE e RAMSDEN, 1983).

Quanto à relevância das pesquisas sobre abordagens à aprendizagem, há evidências de que a utilização da AP influencia positivamente o desempenho acadêmico, contribui para uma aprendizagem efetiva, possibilita a superação de dificuldades pessoais e ambientais, e potencializa a aprendizagem (DA SILVA e SÁ, 1993; GARNER et al., 1984; GOMES, 2011; PRESSLEY e LEVIN, 1983). 


\subsection{Autorregulação da aprendizagem}

A autorregulação da aprendizagem pode ser definida como o processo relacionado aos pensamentos, sentimentos e ações autogerados e planejados de acordo com as necessidades do indivíduo, de modo a influenciar a própria aprendizagem ou motivação e possibilitar a execução de metas pessoais (ZIMMERMAN, 2000). Tal processo pode ser entendido como o controle e a regulação do próprio estudante sobre seus pensamentos, cognição, afeto, motivação, comportamento e ambiente, em prol de objetivos acadêmicos (ROSÁRIO, 2004; VEIGA SIMÃO e FRISON, 2013; ZIMMERMAN, 2013).

Aprender a utilizar estratégias específicas de aprendizagem, como, por exemplo, o estabelecimento de metas, a gestão do tempo, a busca por ajuda ou informações, autoavaliações, constitui uma maneira de organizar e planificar a aprendizagem, antes e durante sua realização, de forma que, na revisão das tarefas, os alunos possam verificar os resultados obtidos.

O ciclo da autorregulação descrito por Zimmerman (2013) está representado em fases, como pode-se observar na a Figura 1.

\section{Figura 1 - Fases da autorregulação da aprendizagem.}

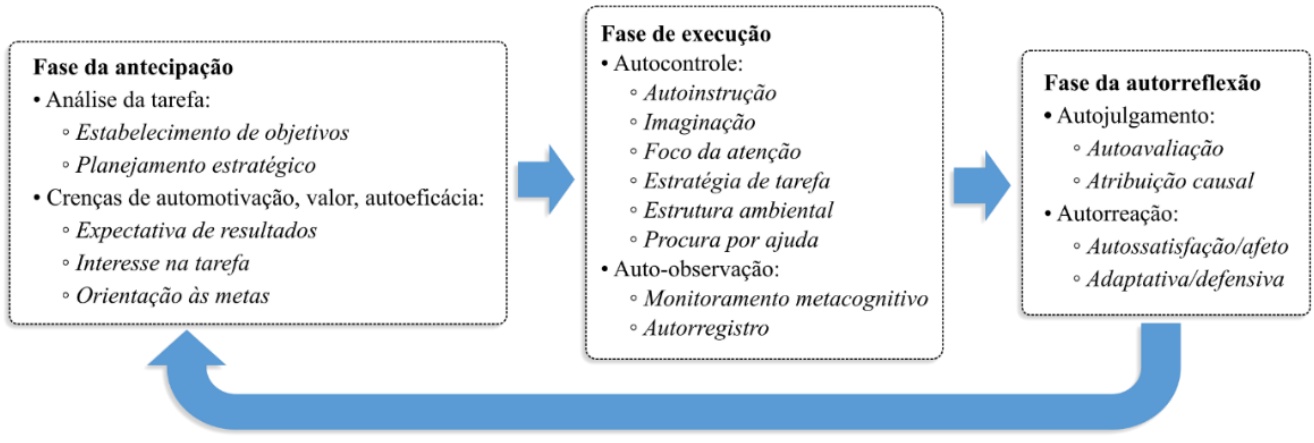

Fonte: Adaptado de (FRISON, 2016).

A autorregulação do aprender é vista como um processo cíclico, dinâmico e integrado, que se retroalimenta pelo feedback das experiências de aprendizagem do próprio aluno (ZIMMERMAN, 2001; ZIMMERMAN, 2013). Ela constitui uma forma de organizar e planificar as estratégias de aprendizagem, tanto antes e durante sua realização, como na revisão das tarefas ou verificação dos resultados.

As fases são dispostas de forma cíclica, estabelecendo relações e conexões entre si; elas se imbricam e se articulam, pois são processos integrados e dinâmicos, e representam como o estudante autorregulado tende a agir. Ao reavaliar seu planejamento, o aluno monitora seus esforços, bem como avalia e altera comportamentos, sentimentos e pensamentos para alcançar as metas estabelecidas (FRISON, 2016).

\subsection{Jogos educacionais}

Jogos educacionais são jogos desenvolvidos com o objetivo de ensinar as pessoas sobre um determinado assunto, conceito ou área de estudo.

Os bons jogos devem incorporar alguns princípios de aprendizagem para que se tornem atrativos e, ao mesmo tempo, transmitam conhecimentos. Esses princípios de aprendizagem, conforme Gee (2004), são listados a seguir:

- Identidade: o usuário se identifica com o jogo e assume um compromisso com ele; 
- Interação: os jogos reagem às ações dos usuários, oferecendo feedbacks e novos problemas;

- Produção: os usuários co-desenham os jogos pelas ações que executam e as decisões que tomam, são ativos no processo;

- Riscos: as consequências das falhas dos jogadores são reduzidas, estimulando-os a correr riscos, explorar;

- Customização: os usuários têm liberdade para customizar um jogo para que ele se ajuste aos seus estilos de aprender e de jogar, respeitando seu ritmo de aprendizado;

- Agência: sensação de agência e controle;

- Boa ordenação dos problemas: estimula os usuários a pensar em soluções criativas para problemas complexos, ordenando-os de acordo com suas competências, habilidades e ritmo próprio;

- Desafio e consolidação: os bons jogos oferecem um conjunto de problemas e os usuários recebem novos desafios apenas quando o conhecimento anterior está consolidado;

- "Na hora certa" e "a pedido": os jogos quase sempre dão as informações quando os usuários precisam delas e quando podem usá-las, otimizando o uso dessas informações;

- Contextualização: colaboram na contextualização de sentidos e significados;

- Frustração prazerosa: os desafios apresentados são "factíveis", mas desafiadores;

- Pensamento sistemático: os jogos encorajam os jogadores a pensar sobre as relações e não sobre eventos, fatos e habilidades isolados;

- Explorar, pensar lateralmente, repensar os objetivos: encorajam os usuários a explorar detalhadamente antes de irem adiante com rapidez, a pensar lateralmente e não só linearmente e a usar essa exploração e esse pensamento lateral para repensar os próprios objetivos;

- Ferramentas inteligentes e conhecimento distribuído: os jogos "emprestam" ferramentas (habilidades e conhecimentos próprios) para que o usuário alcance seus objetivos;

- Equipes transfuncionais: estimulam o trabalho em equipe, onde cada jogados possui funções e habilidades diferentes;

- Performance anterior à competência: permite a aprendizagem prática.

Assim, percebe-se que é possível e desejável que a educação, em seus diversos âmbitos, possa se beneficiar do uso de jogos educacionais como ferramenta de ensino.

\section{METODOLOGIA}

O presente estudo de caso foi desenvolvido a partir de uma revisão bibliográfica que visou determinar o eixo referencial que serve de embasamento teórico para o desenvolvimento do jogo "Plugue". Foram levantadas as principais referências no âmbito da aplicação de jogos educacionais, em especial para o ensino na engenharia, bem como as pesquisas mais relevantes no campo das teorias das abordagens à aprendizagem e da autorregulação da aprendizagem.

Foram traçados paralelos entre as teorias que sustentam o desenvolvimento dos jogos e a teoria da autorregulação da aprendizagem, de modo que fosse possível a implementação de um jogo que contribuísse de forma substancial para o aprendizado dos estudantes ao estimular a adoção de motivações e estratégias profundas de aprendizagem, além de servir como recurso metodológico para docentes. 
A partir da definição do ferramental teórico, foram definidos quais experimentos de um laboratório de instalações elétricas prediais deveriam caracterizar as fases do jogo, bem como definiram-se as ferramentas o jogo deveria ter, e os critérios de avaliação a serem aplicados.

Foram implementados oito experimentos, divididos em oito fases, baseados na NBR 5410-2004 (ABNT, 2004) e no Guia de Instalações Prediais do Curso de Instalações Elétricas da Universidade Federal Campina Grande (COSTA, 2007): Interruptor simples; Interruptor duplo; Tomada simples; Tomada dupla; Interruptor com tomada; Interruptor three-way; Interruptor four-way; Fotocélula.

Visando facilitar a contextualização com a temática do jogo e a identificação do usuário, os cenários escolhidos para as fases do jogo consistem em cômodos de uma residência. $\mathrm{O}$ jogo "Plugue" foi desenvolvido em ambiente Unity 3D (UNITY TECHNOLOGIES, 2020), com artes executadas empregando o editor de imagens vetorizadas InkScape (INKSCAPE, 2020). A metodologia de execução do projeto do jogo foi adaptada a partir do método Scrum (SCRUM, 2020).

\section{RESULTADOS E DISCUSSÕES}

Os critérios propostos por Gee (2004), citados na seção 2.3, foram utilizados para construir o jogo "Plugue", cuja temática é voltada para o ensino de instalações elétricas prediais. Buscou-se desenvolver o jogo de modo que ele contribuísse para o processo mais significativo de aprendizagem dos alunos.

Para tal, realizou-se uma correlação entre as características principais dos jogos educacionais, as fases da autorregulação da aprendizagem e os recursos do jogo "Plugue". Assim, os critérios e funcionalidades implementados no jogo foram baseados na correlação proposta. Estas correlações são apresentadas no diagrama da Figura 2.

Figura 2 - Correlação entre os princípios dos bons jogos, as fases da autorregulação da aprendizagem e o jogo "Plugue".

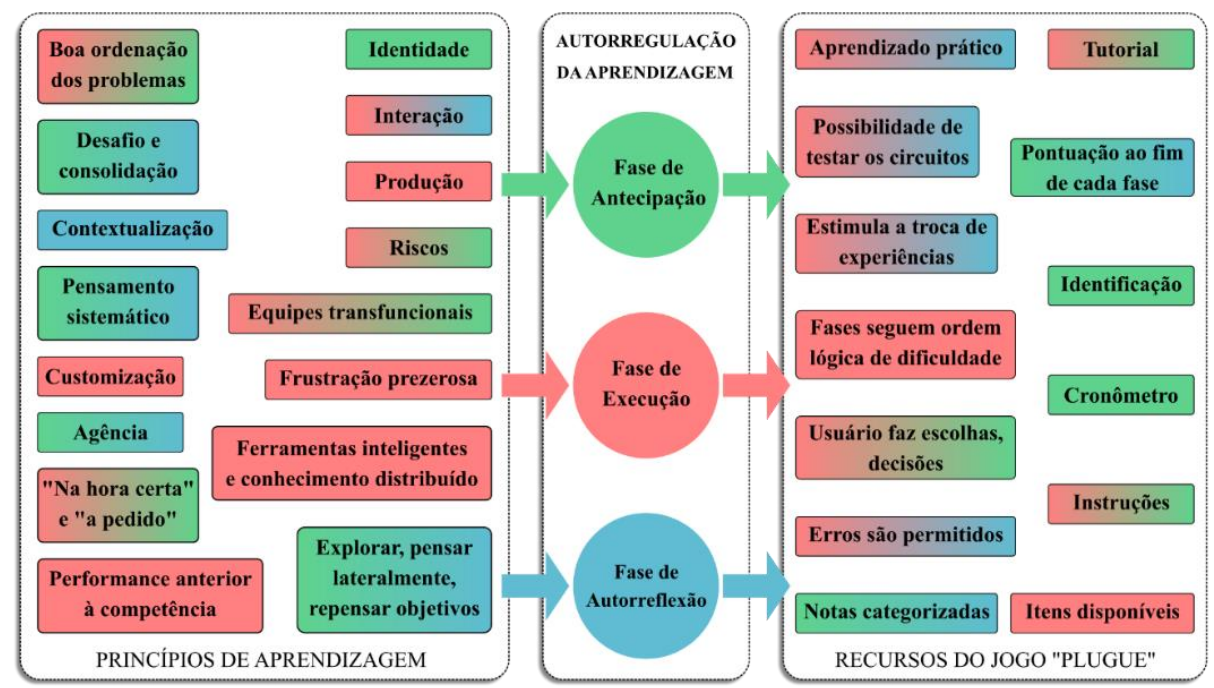

Os princípios dos bons jogos estão relacionados a ações de planejamento frente à atividade a ser realizada ou meta a cumprir; ações de execução, nas quais o usuário faz uso dos recursos e ferramentas do jogo para atingir objetivos; e ações de feedback, onde o usuário verifica seu desempenho. Tais ações podem ser associadas às fases da autorregulação da 
aprendizagem, conforme observado na Figura 2. A partir disso foi possível desenvolver o jogo "Plugue" de modo que sua dinâmica, ferramentas e critérios de avaliação com base nas correlações feitas entre os princípios dos bons jogos e a autorregulação da aprendizagem.

Os critérios propostos por Gee $(2004,2008)$ foram sintetizados por Bomfoco e Azevedo (2012) em cinco fatores: Metas, Curva de aprendizagem, Oportunidades de exploração, Feedback e Colaboração e socialização. As descrições dos fatores estão sendo apresentadas no Quadro 1, bem como as relações estabelecidas entre os fatores, as fases da autorregulação e o jogo "Plugue".

Quadro 1 - Jogo "Plugue" e sua relação com as

fases da autorregulação da aprendizagem e os princípios dos bons jogos.

\begin{tabular}{|c|c|c|c|}
\hline $\begin{array}{c}\text { Fatores } \\
\text { considerados }\end{array}$ & Descrição & $\begin{array}{c}\text { Fase da } \\
\text { Autorregulação }\end{array}$ & Jogo "Plugue" \\
\hline Metas & $\begin{array}{c}\text { As pessoas armazenam melhor suas } \\
\text { experiências quando estão relacionadas } \\
\text { a metas. }\end{array}$ & $\begin{array}{l}\text { Fase de Antecipação: } \\
\text { análise da tarefa, } \\
\text { estabelecimento de } \\
\text { objetivos e metas, } \\
\text { planejamento } \\
\text { estratégico. }\end{array}$ & $\begin{array}{l}\text { 1. Tutorial; } \\
\text { 2. Instruções com objetivos; } \\
\text { 3. Sentimento de pertencimento, } \\
\text { identificação com o contexto } \\
\text { do jogo; } \\
\text { 4. Cronômetro. }\end{array}$ \\
\hline $\begin{array}{l}\text { Oportunidades } \\
\text { de exploração }\end{array}$ & $\begin{array}{l}\text { As experiências devem ser interpretadas } \\
\text { durante e após as ações. Lições devem } \\
\text { ser extraídas das experiências anteriores } \\
\text { a fim de antecipar em quais outros } \\
\text { contextos e de que forma estas lições } \\
\text { podem ser úteis novamente. } \\
\text { Oportunidades para aplicar suas } \\
\text { experiências anteriores em novos } \\
\text { contextos, permitindo a generalização. }\end{array}$ & $\begin{array}{l}\text { Fase de Execução: } \\
\text { autoinstrução, } \\
\text { imaginação, estratégia, } \\
\text { busca ajuda, auto- } \\
\text { observação; execução } \\
\text { do planejamento. }\end{array}$ & $\begin{array}{l}\text { 5. Itens à disposição do usuário; } \\
\text { 6. Fases seguem ordem lógica e } \\
\text { de dificuldade; } \\
\text { 7. Aprendizado prático; } \\
\text { 8. Usuário faz escolhas, toma } \\
\text { decisões; } \\
\text { 9. Erros são permitidos. }\end{array}$ \\
\hline $\begin{array}{c}\text { Colaboração } \\
\text { e Socialização }\end{array}$ & $\begin{array}{l}\text { Feedback imediato durante as suas } \\
\text { experiências para que possam } \\
\text { reconhecer seus erros. É importante que } \\
\text { possam explicar seus erros e o que } \\
\text { poderiam ter feito de forma diferente. } \\
\text { Aprendizado a partir das experiências } \\
\text { de outras, discussão com seus pares e } \\
\text { instrução dada por mentores. }\end{array}$ & $\begin{array}{c}\text { Fase de Autorreflexão: } \\
\text { autoavaliação, verifica } \\
\text { se os objetivos foram } \\
\text { cumpridos. }\end{array}$ & $\begin{array}{l}\text { 10. Feedback; } \\
\text { 11. Pontuação ao fim de cada } \\
\text { fase; } \\
\text { 12. Notas categorizadas; } \\
\text { 13. Possibilidade de testar o } \\
\text { circuito; } \\
\text { 14. Estimula a troca de } \\
\text { experiências entre os usuários. }\end{array}$ \\
\hline
\end{tabular}

A dinâmica do jogo "Plugue" busca estimular o usuário a utilizar as fases da autorregulação da aprendizagem ao permitir que o usuário tenha acesso aos objetivos do jogo de forma clara e estabeleça metas de acordo com as atividades a serem feitas (fase de antecipação); ao disponibilizar acessórios, estimular a tomada de decisões e permitir erros durante e para o cumprimento das tarefas (fase de execução); e ao permitir a testagem dos circuitos e fornecer feedback imediatamente após cada estágio, permitindo que o usuário verifique seus erros e corrija-os nos estágios posteriores (fase de autorreflexão).

Cada uma das principais características, numeradas de 1 a 14 no Quadro 1, estão sendo mostradas nas Figuras 3, 4 e 5, de acordo com as fases da autorregulação às quais se associa. As imagens apresentadas nas Figuras 3 a 5 são quadros capturados do próprio jogo.

A correlação proposta foi fundamental para a implementação do jogo, desde os cenários utilizados, através dos quais o usuário se identifica com o jogo e se torna capaz de fazer generalizações do conhecimento adquirido; até a forma como o usuário é avaliado, visto que é importante estimular o aluno a refletir sobre suas decisões com base no seu aprendizado, além de permitir e minimizar erros com o objetivo de estimular a exploração por parte do usuário. 
Espera-se também que o jogo contribua para a interação aluno-professor e aluno-aluno, fundamental para que o processo de ensino-aprendizagem ocorra de forma efetiva.

Figura 3 - Autorregulação da aprendizagem e o jogo

"Plugue": Fase de Antecipação da Autorregulação da Aprendizagem.

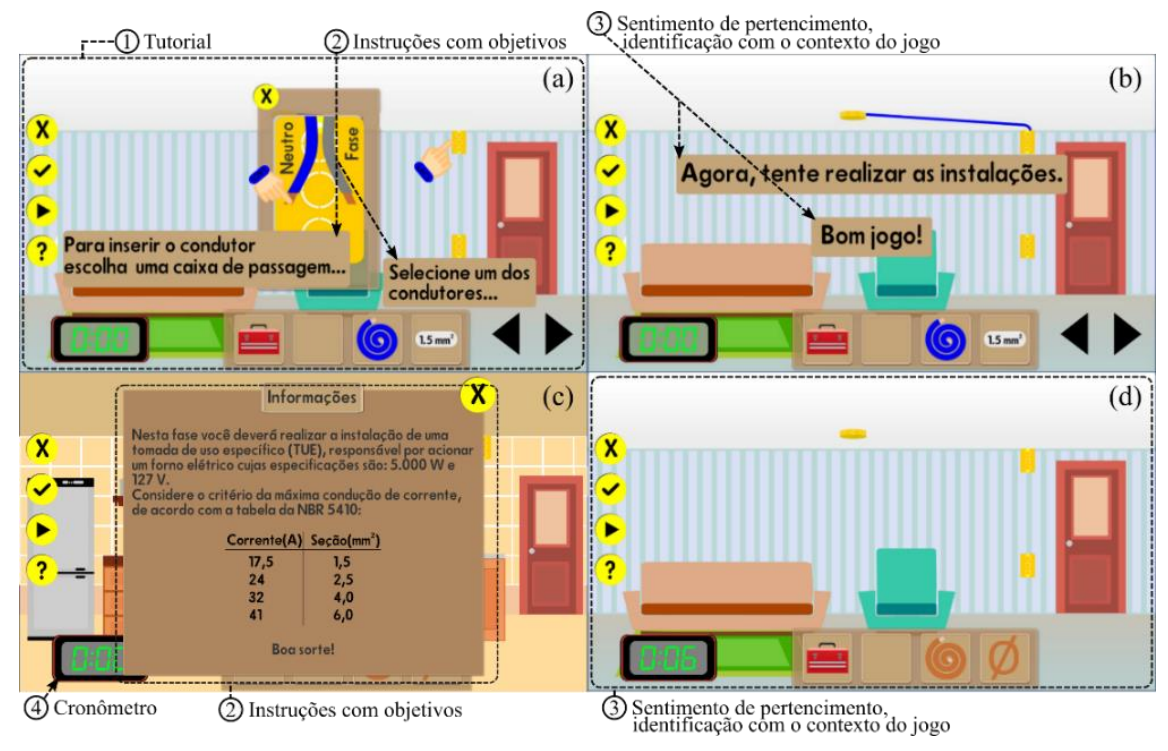

Figura 4 - Autorregulação da aprendizagem e o jogo "Plugue": Fase de Execução da Autorregulação da Aprendizagem.

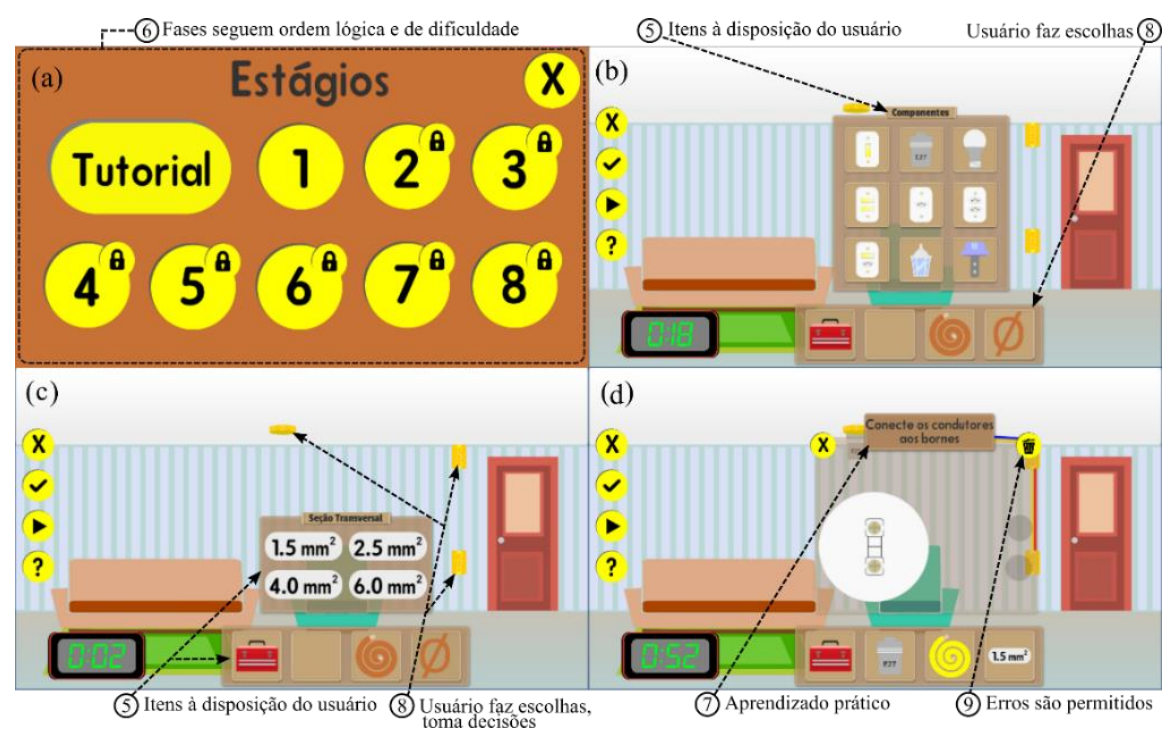


"Os desafios para formar hoje o engenheiro do amanhã"

BIGGS, J. B. The Study Process Questionnaire (SPQ): Manual. Hawthorn, Vic.: Australian Council for Educational Research, 1987b.

BIGSS, J. B. Student Approaches to Learning and Studying. Hawthorn, Vic.: Australian Council for Educational Research, 1987a.

BOMFOCO, M. A.; AZEVEDO, V. D. A. Os jogos eletrônicos e suas contribuições para a aprendizagem na visão de J. P. Gee. CINTED-UFRGS Centro Interdisciplinar de Novas Tecnologias na Educação, Porto Alegre, 2012.

BORUCHOVITCH, E. Estratégias de aprendizagem e desempenho escolar: Considerações para a prática educacional. Psicologia: Reflexão e Crítica, v.12, n.2, p. 361-373, 1999.

COSTA, E. G., SOUZA, R. T., FERREIRA, T. V. Guia de Instalações Prediais Elétricas, Grupo de Sistemas Elétricos, Laboratório de Alta Tensão, Universidade Federal de Campina Grande, 2009.

DRAW Freely. Inkscape. Disponivel em: https://inkscape.org/. Acesso em: 18 maio 2020.

DA SILVA, A. L.; SÁ, I. Saber estudar e estudar para saber: Ciências da Educação. Porto, Portugal: Porto. 1993.

ENTWISTLE, N. J.; RAMSDEN, P. Understanding student learning. London: Croom Helm, 1983.

FRISON, L. M. B. Autorregulação da aprendizagem: abordagens e desafios para as práticas de ensino em contextos educativos. Revista Educação PUC - Campinas, v. 21, n.1, p. 1-17, 2016.

GARNER, R.; HARE, V. C.; ALEXANDER, P. HAYNES, J.; WINOGRAD, P.Inducing use of a text lookback strategy among unsucessful readers. American Educational Research Journal, v. 21, p. 789-798, 1984.

GEE, J. P. What video games have to teach us about learning and literacy. Nova York: Palgrave Macmillan, 2004.

GEE, J P. Video Games, Learning, and "Content". In: Miller, Christopher Thomas Purpose and Potential in Education. Nova York: Springer, 2008

GOMES, C.M.A.; GOLINO, H.F.; PINHEIRO, C.A.R.; MIRANDA, G.R.; SOARES, J. Validação da Escala de Abordagens de Aprendizagem (EABAP) em uma amostra brasileira. Psicologia: Reflexão e Crítica, v. 24, n.1, p. 19-27, 2011.

MARTON, F; SÄLJÖ, R. On qualitative differences in learning: I - Outcome and Process. British Journal of Educational Psychology, v. 46, p. 4-11, 1976a.

MARTON, F; SÄLJÖ, R. On qualitative differences in learning: II - Outcome as a function of the learner's conception of the task. British Journal of Educational Psychology, v. 46, p. $115-127,1976 b$. 
"Os desafios para formar hoje o engenheiro do amanhã"

PRESSLEY, M.; LEVIN, J. R. Cognitive strategy research: Psychological Foundations. New York: Springer-Verlag, 1983.

ROSÁRIO, P.; ALMEIDA, L.; NÚÑEZ, J. C.; GONZÁLEZ-PIENDA, J. A. Abordagem dos alunos à aprendizagem: Análise do construto. Psico-USF, v.9, n.2, p. 117-127, 2004.

SCRUM. The Home of Scrum: What is Scrum? Disponível em: https://www.scrum.org/resources/what-is-scrum. Acesso em 18 de maio de 2020.

UNITY. Unity Technologies. Disponível em: https://unity.com/pt. Acesso em: 18 maio 2020.

VEIGA SIMÃO, A. M.; FRISON, L.M. Autorregulação da aprendizagem: abordagens teóricas e desafios para as práticas em contextos educativos. Cadernos de Educação, v.45, p.2-20, 2013.

ZIMMERMAN, B. J. Theories of self-regulated learning and Academic achievement: An overview and analysis. In: Zimmerman, B.; Schunk, D. Self-regulated learning and academic achievement: Theoretical perspectives. New York: Lawrence Erlbaum Associates, p. 1-37, 2001.

ZIMMERMAN, B.J. Becoming a self-regulated learner: An overview. Theory into Pratice, v.41, n.2, 64-70, 2002.

ZIMMERMAN, B. J. From cognitive modeling to self-regulation: a social cognitive carrier path. Educational psychologist, v. 48, n.3, p. 135-147, 2013.

\title{
LEARNING SELF-REGULATION AND THE EDUCATIONAL SOFTWARE DEVELOPMENT: A CASE STUDY
}

\begin{abstract}
In the context of engineering education, it is already possible to understand the importance of using technological resources capable of stimulating the adoption of a collaborative, active teaching model, capable of helping students to develop the necessary skills to obtain professional success in a society in constant evolution. Among the technological educational tools, the games stand out for being able to benefit several aspects of the student, among them the affective, social and cognitive aspects, in addition to enabling the development of characteristics such as imagination, imitation, the rule, decision-making and logical reasoning. In this work, we propose to present an approach that relates the theory of learning self-regulation to the learning principles incorporated by games. It is hoped that the proposed approach can provide subsidies for the development of interactive educational software, active in the learning process, and that stimulate the adoption of motivations and strategies related to deep learning, contributing to the development of cognitive aspects of the student. As a case study, the educational software "Plugue" is presented, developed for teaching building electrical installations, which used the proposed approach as a reference axis for its construction and its form of intervention in the learning process.
\end{abstract}

Keywords: Engineering, education, self-regulation of learning, approaches to learning, educational games. 